\title{
$\begin{array}{ll}\text { Research Square } & \text { Preprints are preliminary reports that have not undergone peer review. } \\ \text { They should not be considered conclusive, used to inform clinical practice, }\end{array}$
}

\section{Effect of serum bisphenol A and low T3 on short-term prognosis of young patients with intracerebral hemorrhage}

guoping wang ( $\sim$ guopingwang60a@163.com )

Affiliated Changzhi People's Hospital of Shanxi Medical University https://orcid.org/0000-0002-7520-4345

Jinhua Chen

Affiliated Changzhi People's Hospital of Shanxi Medical University

Zhongdong Qiao

Central Laboratory,Shanghai Jiao Tong University, Institute of Agricultural and Biological

Lili Zhao

Affiliated Changzhi People's Hospital of Shanxi Medical University

\section{Dongxing Guo}

Shanxi Medical University Basic medical college

\section{Ping Guo}

Affiliated Changzhi People's Hospital of Shanxi Medical University

\section{Aiven Wang}

Affiliated Changzhi People,s Hospital of Shanxi Medical University

Wanli Sun

Changzhi Medical College Peace Hospital

Lifang Zhang

Changzhi Medical College Peace Hospital

Cueiping Feng

Changzhi Second People, s Hospital of Shanxi Provincial

Jiyuan Lyu

First Hospital of Shanxi Medical University

\section{Research Article}

Keywords: Bisphenol A, Young patient, Intracerebral hemorrhage, Low T3 syndrome, Sub-clinical hypothyroidism, miRNA-451/miRNA338-3p, Biomarker

Posted Date: November 8th, 2021

DOI: https://doi.org/10.21203/rs.3.rs-1058244/v1

License: () (1) This work is licensed under a Creative Commons Attribution 4.0 International License. Read Full License 


\section{Abstract}

Background

Bisphenol A (BPA) is widely present in daily necessities, and its exposure is almost everywhere. We aimed to evaluate the relationship between serum BPA levels, biomarkers and recent mortality in young patients with intracerebral hemorrhage (YICH) and low T3 .

Methods

Using a multi-center longitudinal prospective study, the research team recruited $600 \mathrm{YICH}$ patients and divided into two subgroups according to their thyroid function, of which 320 (53.3\%) were in the LT3, Sub-clinical hypothyroidism (SCH) was $280(46.6 \%)$ and control group $(n=600)$.All patients received serum BPA, and biomarker blood draws.

Results

Calculating the cumulative quantification of plastic bag products, BPA increased by 0.204 standard deviation unit per log unit ( $<<0.003)$. Serum BPA, thrombin and matrix metalloproteinase (MMP-9) \{BPA $(0.91 \pm 0.14)$ vs $(0.765 \pm 0.13)$ vs. $(0.535 \pm 0.11) \mathrm{pg} / \mathrm{ml}$, thrombin $(1.35 \pm 0.18)$ vs $(0.66 \pm) 0.15)$ vs $(0.66 \pm 0.15) \mathrm{ng} / \mathrm{ml}$, MMP-9 $(104 \pm 10$ vs $(53 \pm 8.1)$ vs $(53 \pm)$ 8.1) pg/ml, both $\mathrm{P}<0.01\}$. Linear correlation analysis showed that serum BPA was positively correlated with miR-388-3p and 24hDBPCV, and negatively correlated with LT3 and miR-451. Multiple linear regression showed that BPA affected the increase of $n D B P$ Percentage of independent risk factors $(\beta=0.286), P<0.01$. Mortality rate showed 2,15 and 30 days of patients in the LT3 and the SCH of $4.7 \%$ vs $2.5 \%, 6.25 \%$ vs $3.75 \%, 4.68 \%$ vs $2.5 \%, P<0.01$.

Conclusion

High BPA exposure strongly predicts the risk of $\mathrm{ICH}$ in young patients. The activation of NF-kB signaling pathway, secondary thyroid function, ambulatory blood pressure scores, and changes in messenger ribonucleic acid construct an inflammatory response system. There are also significant differences in recent mortality between the LT3 group and the SCH group.

\section{Trial registration}

The trial is registered at clinical .gov (www.medresman.Chi CTR1900023626)

\section{Highlights}

1. Our study proved for the first time that serum BPA levels in young patients with intracerebral hemorrhage (YICH) are significantly increased, which may be a key and new finding.

2. There are obvious gender differences in serum bisphenol A levels in YICH patients, and this difference is related to the significant decrease in serum testosterone levels of functional hormones caused by the body's stress response.

3. It reveals the obvious inflammatory response after cerebral hemorrhage, the activation of cytokines, and the formation of a complete inflammatory network system, from biological agents to cytotoxicity and gene toxicity. Shows the difference in the temporal expression of biomarkers .

4. In BPA-related YICH patients, there are changes in ambulatory blood pressure (especially nocturnal diastolic blood pressure, blood pressure score), gender differences in thyroid function stress changes, and the combined effect of long-term accumulation of BPA.

5. In patients with IYICH, there is a significant difference in the recent mortality between the low T3 group and the sub-clinical hypothyroidism group.

\section{Introduction}

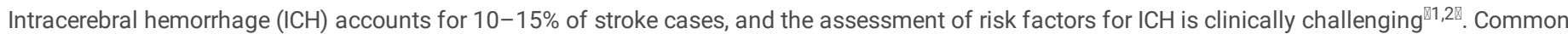
mutations in gene locus, vascular risk factors (hypertension or blood pressure variability), peripheral gene toxicity and dynamic changes of immune cells are closely related to the risk of stroke ${ }^{\llbracket 3,4 \rrbracket}$. For this reason, exploring the unique field of interaction between self-protective neuroimmune inflammation and messenger ribonucleic acid will likely bring strategic positioning to the long-term prognosis or treatment of ICH ${ }^{85 \mathbb{R}}$. This study focuses on the changes in the levels of bio-trigger BPA gene toxicity and neurotoxicity and inflammation markers, in order to further evaluate the relationship between multiple proteases targeted in ICH and BPA, which will help patients with cerebral hemorrhage Neurotoxic molecular research provides in-depth new insights.

BPA is an industrial chemical that exists widely in the environment. Humans are exposed to BPA through food, milk, and skin contact ${ }^{[6,7]}$. High levels of BPA exposure are related to abnormal regulation of thyroid , autoimmune functions, obesity and metabolism ${ }^{[8,9]}$. However, at current exposure levels in developing world the link between $\mathrm{ICH}$ and BPA remain unproven. This study focuses on clarifying the relationship between the cumulative quantification of BPA exposure levels and the risk of $\mathrm{ICH}$, and assessing the correlation between different thyroid function,ambulatory blood pressure (ABP),miRNA,biomarkers and recent mortality.

\section{Material And Methods}




\section{Patient}

Analyze the clinical data of 600 YICH patients admitted to the Department of Intensive Care and Neurology,Changzhi People's Hospital. Changzhi Medical College Peace Hospital and Changzhi Second People's Hospital from January 2011 to January 2014. All selected cases are used Systematic sampling, multicenter and randomized controlled studies. According to the level of thyroid function, they were divided into two subgroups, including 320 (53.3\%) in the LT3 and $280(46.6 \%)$ in the $\mathrm{SCH}$. Meets the criteria of the American Stroke Association,Clinical Neurology Committee $\underline{010}$. Exclusion criteria: (1) Liver and kidney damage, visceral malignant tumors, history of diabetes, family history of hypertension and previous use of aspirin. (2) Patients with cerebrovascular malformations and cerebrovascular tumors found in computed tomography must be excluded through cerebrovascular angiography.

The normal control group is selected strictly according to the screening criteria: a history of long-term exposure to plastic products are not eligible, healthy individuals $(n=600)$ matching age and gender .

\section{Follow-up data}

The methods include household entry, community visits, telephone calls, and reporting. Investigation of long-term exposure to plastic products: understand the use of plastic bottles from the time of infancy, children, adolescents, and adults who have habitually contacted or used plastic bags, especially the fried dough sticks were taken out of the oil pan and put into the plastic bag for breakfast. The establishment of product quantitative. Additionally, collect routine life data, including patient medication history, smoking and drinking history, body mass index (BMI): BMI = weight $(\mathrm{kg}) / \mathrm{height}^{2}\left(\mathrm{~m}^{2}\right)$. Smoking is defined as continuous cumulative smoking for 6 months or more; drinking is defined as drinking at least one glass of wine in the past 30 days .

\section{4h ABP monitoring}

Strictly follow the 24-hour ABP inspection precautions and standards, and use the following measurement parameters:24hSBP®24hDBP『dSBP, dDBP;nSBP, $\mathrm{nDBP}$; BP variability and coefficient of variation (BPV,CV), 24h SBPCV, 24hDBPCV; dSBPCV, dDBPCV; $\mathrm{nSBPCV}$, nDBPCV;The coefficient is equal to the standard deviation/ average BP.The diagnostic criteria for hypertension are $\mathrm{SBP} \geq 140$ and $\mathrm{DBP} \geq 90 \mathrm{mmHg}(1 \mathrm{mmHg}=0.133 \mathrm{kpa})^{\square 110}$.

\section{Serum analysis}

Serum samples were collected on an empty stomach in the morning on the 2, 15 and 30 days after admission. The enzyme-linked immunosorbent assay measures BPA, thrombin, and MMP-9 from Shenzhen Galvanomi Biotechnology Co,Ltd.Provided; Electrochemi luminesc- ence immunoassay measurement of total testosterone and free testosterone are provided by Bayer,triiodothyroxine (T3) and free triiodothyroxine(FT3), thyroxine (T4), free thyroxine (FT4) and Thyroid Stimulating Hormone (TSH) provided by Shanghai Science Co, Ltd.

\section{TLR4-NF-KB signaling pathway related protein expression:}

Collect peripheral venous blood, separate mononuclear cells, use Western Blot method to detect, Toll-like receptor 4 (TLR-4) , Myeloid differentiation factor 88 (My88),(TRAF6)tumor necrosis factor-associated factor6, nuclear transcription factor KBp65 (NF-kBp65). The protein was extracted from mononuclear cells with RIPA lyase buffer containing protease inhibitors, and the color was developed using a chemiluminescence kit. Perform quantitative analysis on the image and calculate the relative expression of each protein.

\section{Expression of miRNA338-3p and miRNA144/451 in peripheral blood lymphocytes}

Take $2 \mathrm{ml}$ of fasting venous blood was drawn from the survey subjects, and total RNA was extracted by the Trizol method. Real-time fluorescence quantitative polymerase chain reaction (PCR) method to detect the expression level of miRNA: using total RNA as a template, using random primers for reverse transcription amplification synthesis,obtaining miR-338-3p,miR144/451 internal reference Gene glyceraldehy-de 3-phosphate dehydrogenase (GAPDH) RNA d cDNA template; specific PCR primers were used to detect the expression of miRNA and GAPDH genes, and the 2- $\triangle \triangle \mathrm{ct}$ method was used to analyze the expression of indicators.

\section{Statistical analysis}

General demographic data is the mean \pm SD of continuous variables and the percentage of categorical variables. The qualitative data comparison adopts oneway analysis of variance and chi-square test, and the main statistical indicators are tested for normality. First, the Mann-Whitney $\mu$ test is used to assess the average difference between the two groups. The comparison of the means of the two large samples of the group design uses the $\mu$ test. The comparison of the three sets of data uses F test. The changes of serum BPA concentration, cytokines, protease and $24 \mathrm{hBP}$ were analyzed by linear correlation analysis, regression analysis was used to determine the correlation between BPA concentration and thyroid function ; through multiple linear regression analysis, the influence of various factors on the percentage of nSBP and nDBP was analyzed. Use receiver operating characteristic curve (receiver operating characteristic curve, ROC curve) to define the boundary value of miRNA high and low expression level; use cross-sectional analysis combined with Logistic regression model to analyze the combination of serum BPA, ABP and miRNA expression levels, cross-effects, and calculate relative Risk ratio attribution ratio and interaction index. Using SPSS v20.0 software (SPSS Inc, USA) for statistical analysis, the difference was statistically significant, $P<0.05$.

\section{Results}


There were 240 males and 80 females in the LT3 group (75\% vs 25\%); 80 males and 200 females in the SCH group (28,57\% vs $71.42 \%)$; the average age was $25 \pm 7.2$ years, and the control group was $25.3 \pm 6.6$ years old. Compared with the control group, $24 \mathrm{hSBP}, 24 \mathrm{hDBP}$, insulin resistance-index, blood lipids, etc. increased in all $\mathrm{YICH}$ patients, especially $\mathrm{nDBP}$ increased significantly, $\mathrm{P}<0.05$. Additionally, calculating the cumulative amount of plastic bags for 10 years, each bag is 1.6 grams, 3000 bags, the total is about $4800 \mathrm{~g}$. For every log unit increase in BPA, it is determined that the $Z$ score of the plastic bag increases by 0.204 standard deviation units $(\mathrm{P}<0.003)$. (Table 1$)$ 
Table 1

Characteristics of the population

\begin{tabular}{|c|c|c|c|c|}
\hline variable & LT3 $(n=320)$ & $\operatorname{SCH}(n=280)$ & Control $(n=300)$ & $P$ value \\
\hline Mean age, (range) & $25.4 \pm 4.88$ & $25.5 \pm 6.29$ & $25 \pm 7.285$ & 0.39 \\
\hline Male, n (\%) & $280(87.5) * *$ & $80(28.57)$ & $300(50)$ & 0.05 \\
\hline Married n (\%) & $218(68.1)$ & $165(58 . .9)$ & $300(62.5)$ & 0.20 \\
\hline $\mathrm{BMI}(\mathrm{kg} / \mathrm{m} 2)$ & $23.7 \pm 4.1^{*}$ & $23 \pm 5.1^{*}$ & $21.1 \pm 3.3$ & 0.05 \\
\hline $\mathrm{FPG}(\mathrm{mmol} / \mathrm{L})$ & $5.8 \pm 1.24^{\star \star}$ & $5.2 \pm 0.9 *$ & $4.8 \pm 0.8$ & 0.05 \\
\hline HOMA-IR, (g/L) & $3.5 \pm 1.2^{\star *}$ & $3.2 \pm 1.3^{*}$ & $2.6 \pm 1.2$ & 0.05 \\
\hline 24hDBP $(\mathrm{mmHg})$ & $86 . \pm 18.8^{\star \star}$ & $82 \pm 9.1^{*}$ & $69 \pm 6.3$ & 0.01 \\
\hline $\mathrm{nDBP}(\mathrm{mmHg})$ & $86 \pm 15^{\star *}$ & $82 \pm 11^{*}$ & $70 \pm 14$ & 0.01 \\
\hline $\mathrm{TG}(\mathrm{mmol} / \mathrm{L})$ & $1.61 \pm 0.8^{*}$ & $1.4 \pm 0.9$ & $1.27 \pm 0.9$ & 0.05 \\
\hline Tch (mmol/L) & $5.23 \pm 1.9 * *$ & $4.8 \pm 1.4^{*}$ & $3.8 \pm 1.4$ & 0.01 \\
\hline HDL-C (mmol/L) & $0.98 \pm 0.09 *$ & $1.1 \pm 0.2$ & $1.17 \pm 0.3$ & 0.06 \\
\hline LDL-C (mmol/L) & $3.34 \pm 1.1^{* *}$ & $3.0 \pm 0.44^{\star}$ & $2.7 \pm 0.74$ & 0.01 \\
\hline Drinking history $(n, \%)$ & $41(12.8)$ & $30(10.7)$ & $37(9.7)$ & 0.06 \\
\hline \multicolumn{5}{|l|}{ Region(n,\%) } \\
\hline City & $200(62.5)^{\Lambda \Lambda}$ & $200(62.5))^{\Delta \Lambda}$ & $294(61.25)$ & 0.37 \\
\hline Country & $120(37.5)$ & $80(37.5)$ & 186(38.75) & 0.30 \\
\hline \multicolumn{5}{|l|}{ Education level(n,\%) } \\
\hline Primary school & $35(10.9)^{\star}$ & $18(6.42)$ & $20(6.6)$ & 0.06 \\
\hline Secondary School & $275(85.9)^{\wedge} \boldsymbol{\Lambda}$ & $250(89.28)^{\boldsymbol{\Lambda} \Lambda}$ & $270(90)^{\Lambda \Lambda}$ & 035 \\
\hline University or high & $10(3.125)^{\star}$ & $12(4.28)$ & 10(3.33) & 0.37 \\
\hline \multicolumn{5}{|l|}{ Economic status(n,\%) } \\
\hline poor & $20(6.25)$ & $30(9.38)^{*}$ & $16(5.3)$ & 0.17 \\
\hline Medium & $260(81.25 .)^{\Lambda}$ & $200(71.4)$ & $252(84)$ & 0.18 \\
\hline well-off & 40 (12.5) & $50(17.85)$ & $32(10.6)$ & 0.05 \\
\hline \multicolumn{5}{|l|}{ profession(n,\%) } \\
\hline Individual & $230(71.8)^{\Lambda \Lambda}$ & $191(68 . .2)^{\Lambda \Lambda}$ & $220(73.3)^{\wedge \Lambda}$ & 0.37 \\
\hline Cadre & $34(10.6)^{\Lambda \Lambda}$ & $33(11.78)^{\Lambda \Lambda}$ & $30(10)^{\Lambda \Lambda}$ & 0.41 \\
\hline Doctor & $7(2.18)$ & $6(2.14)$ & $5(1.66)$ & 0.45 \\
\hline Teacher & $12(3.75)$ & $10(3.57)$ & $10(3.3)$ & 0.42 \\
\hline Student & $37(11.56)$ & $40(14.28)$ & $35(11)$ & 0.35 \\
\hline \multicolumn{5}{|l|}{ Medications n, (\%) } \\
\hline Cytochrome C & $300(93.8)$ & $276(98.6)$ & - & 0.25 \\
\hline Coenzyme A & $310(96.9)$ & $266(95)$ & - & 0.45 \\
\hline $\mathrm{CBPHI}$ & $277(86.6)^{* *}$ & $186(66.4)$ & - & 0.01 \\
\hline Glycerol fructose & $276(86.3)$ & $228(81.4)$ & - & 0.44 \\
\hline Ulinastatin & $67(29) * *$ & $16(5)$ & - & 0.01 \\
\hline Naloxone & $21(7.5)^{* *}$ & $9(3.2)$ & - & 0.01 \\
\hline
\end{tabular}

Compared with the control, the levels of serum BPA, various proteases, inflammatory cytokines and miR-338-3p on day 2 of LT3 and SCH increased, miR-455 decreased, and blood pressure parameters increased, $P<0.05$. The ratio of free testosterone in male patients to the control group was significantly lower $P$ $<0.01$. In addition, there was no significant difference in serum rT3 and miR-144 levels between the two groups, P $\geq 0.05$, omitted. (Table 2)

Page 5/17 
Table 2

Comparison of serum BPA,biomarkers and BP scores in different gender subgroups.

\begin{tabular}{|c|c|c|c|c|c|c|c|c|}
\hline Paramet & LT3 & & & & $\mathrm{SCH}$ & & & \\
\hline Biomarbimarker & Male $(n=240)$ & Control $(n=240)$ & Female $(n=80)$ & Control $(n=80)$ & Male $(n=80)$ & Control $(n=80)$ & Female $(n=200)$ & Control $(n=200$ ), \\
\hline $\begin{array}{l}\text { BPA,b BPA } \\
\mathrm{ng} / \mathrm{ml} \\
\text { (2) }\end{array}$ & $0.93 \pm 0.18 * * \square$ & $0.52 \pm 0.14$ & $0.79 \pm 0.14$ ** & $0.52 \pm 0.12$ & $0.82 \pm 0.11^{\star \star \square}$ & $0.53 \pm 0.10$ & $0.70 \pm 0.1$ ** & $0.53 \pm 0.1$ \\
\hline MMP-9 ,ng/ I & $109 \pm 11 * \star$ 眈 & $47 \pm 8$ & $99 \pm 9.5^{\star \star}$ & $43 \pm 6$ & $102 \pm 8.5^{\star \star}$ & $43 \pm 6.5$ & $96 \pm 7.8 * \star$ & $43 \pm 6.3$ \\
\hline $\begin{array}{l}\text { Thrombin, } \\
\mathrm{ng} / \mathrm{mL}\end{array}$ & $1.5 \pm 0.5^{\star \star}$ & $0.65 \pm 0.1$ & $1.31 \pm 0.49 * *$ & $0.64 \pm 0.09$ & $1.29 \pm 0.23$ ** & $0.63 \pm 0.1$ & $1.29 \pm 0.23^{\star \star}$ & $0.66 \pm 0.12$ \\
\hline $\begin{array}{l}\mathrm{miR}-338-3 \mathrm{p} \\
\mathrm{ng} / \mathrm{mL}\end{array}$ & $3.1 \pm 0.9 * *$ & $1.2 \pm 0.8$ & $2.8 \pm 0.94 \star \star$ & $1.23 \pm 0.85$ & $2.6 \pm 0.7 \star \star$ & $1.2 \pm 0.51$ & 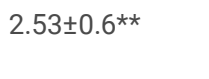 & $1.18 \pm 0.4$ \\
\hline $\begin{array}{l}\mathrm{miR}-451 \\
\mathrm{ng} / \mathrm{mL}\end{array}$ & $2.62 \pm 0.17^{\star *}$ & $3.3 \pm 0.32$ & $2.7 \pm 0.2^{\star \star}$ & $3.2 \pm 0.4$ & $2.83 \pm 0.2^{\star \star}$ & $3.3 \pm 0.5$ & $2.91 \pm 0.14^{\star \star}$ & $3.22 \pm 0.6$ \\
\hline TLR-4,ng/mL & $1.41 \pm 0.21 * * \pi$ & $0.57 \pm 0.10$ & $1.1 \pm 0.2^{\star \star}$ & $0.57 \pm 0.09$ & $1.22 \pm 0.2^{\star \star}$ & $0.53 \pm 0.1$ & $1.06 \pm 0.13^{\star \star}$ & $0.55 \pm 0.2$ \\
\hline MyD88,ng/mL & $\begin{array}{l}1.13 \pm 0.33 \\
\star \star \star \square[0\end{array}$ & $0.44 \pm 0.09$ & $1.1 \pm 0.25^{\star \star}$ & $0.52 \pm 0.1$ & $\begin{array}{l}0.92 \pm 0.1 \\
* *\end{array}$ & $0.49 \pm 0.1$ & $\begin{array}{l}0.90 \pm 0.13 \\
\star *\end{array}$ & $0.45 \pm 0.1$ \\
\hline TRAF6,ng/mL & $\begin{array}{l}1.64 \pm 0.41 \\
\star * \pi[0]\end{array}$ & $0.34 \pm 0.10$ & $\begin{array}{l}1.45 \pm 0.50 \\
\star * \text { 매 }\end{array}$ & $0.33 \pm 0.09$ & $\begin{array}{l}1.42 \pm 0.44 \\
* * \text { 매 }\end{array}$ & $0.36 \pm 0.098$ & $\begin{array}{l}1.37 \pm 0.40 \\
\star * \pi[0]\end{array}$ & $0.35 \pm 0.09$ \\
\hline $\begin{array}{l}\text { NF-kBP } \\
65, \mathrm{ng} / \mathrm{mL}\end{array}$ & $1.21 \pm 0.2^{\star * स[\square] ~}$ & $0.51 \pm 0.10$ & $1.08 \pm 0.2^{\star \star}$ & $0.50 \pm 0.08$ & $1.0 \pm 0.2^{\star \star}$ & $0.48 \pm 0.1$ & $0.86 \pm 0.1 \star \star$ & $0.50 \pm 0.2$ \\
\hline $\mathrm{FT} 3, \mathrm{pg} / \mathrm{ml}$ & $0.74 \pm 0.2 * \star \star \square]$ & $4.8 \pm 0.8$ & $0.86 \pm 0.5^{\star \star \square}$ & $4.8 \pm 0.9$ & $4.17 \pm 0.7$ & $4.33 \pm 0.6$ & $4.2 \pm 0.5$ & $4.8 \pm 1.0$ \\
\hline FT4,pmol/I & $24 \pm 7.2$ & $30 \pm 9.2$ & $26 \pm 9.0$ & $31 \pm 9.2$ & $28 \pm 8.6$ & $28.5 \pm 6.1$ & $26 \pm 12.2$ & $31 \pm 9$ \\
\hline T3, nmol/l & $1.67 \pm 1.0$ & $2.0 \pm 0.6$ & $1.65 \pm 0.9$ & $2.0 \pm 0.6$ & $2.1 \pm 0.5$ & $2.2 \pm 0.4$ & $1.69 \pm 1.0$ & $2.1 \pm 0.7$ \\
\hline $\mathrm{T} 4, \mathrm{nmol} / \mathrm{l}$ & $66 \pm 19.7^{*}$ & $85 \pm 11.4$ & $68 \pm 17.7 *$ & $85 \pm 12$ & $78 \pm 10.7$ & $86 \pm 14$ & $79 \pm 19$ & $85 \pm 11$ \\
\hline $\mathrm{TSH}, \mu \mathrm{IU} / \mathrm{ml}$ & $3.6 \pm 0.4$ & $3.7 \pm 0.7$ & $3.8 \pm 0.6$ & $3.7 \pm 0.9$ & $12.9 \pm 2.18^{\star \star}$ & $3.91 \pm 0.9$ & $9.6 \pm 0.7^{* *}$ & $3.7 \pm 0.5$ \\
\hline $\mathrm{FT}(\mathrm{nmol} / \mathrm{L})$ & $0.13 \pm 0.0 * *$ & $021 \pm 0.05$ & $0.20 \pm 0.06$ & $0.21 \pm 0.05$ & $0.15 \pm 0.05^{\star}$ & $0.20 \pm 0.5$ & $0.19 \pm 0.02$ & $021 \pm 0.04$ \\
\hline $\mathrm{TT}(\mathrm{nmol} / \mathrm{L})$ & $4.9 \pm 1.5^{\star \star}$ & $17 \pm 4.6$ & $16 \pm 4.2$ & $17 \pm 4.6$ & $7 \pm 1.4^{*}$ & $16 \pm 3.5$ & $15 \pm 4.5$ & $17 \pm 4.6$ \\
\hline $\begin{array}{l}24 \mathrm{hDBPCV} \\
\text { (95\%,interval) }\end{array}$ & $\begin{array}{l}0.17(0.12- \\
0.19)^{\star \star}\end{array}$ & $\begin{array}{l}0.11(0.08- \\
0.12)\end{array}$ & $\begin{array}{l}0.16(0.12- \\
0.19)^{\star \star}\end{array}$ & $\begin{array}{l}0.10(0.08- \\
0.11)\end{array}$ & $\begin{array}{l}0.14(0.07- \\
0.15)^{\star}\end{array}$ & $\begin{array}{l}0.09(0.06- \\
0.10)\end{array}$ & $0.13(0.1-0.16)^{\star}$ & $\begin{array}{l}0.09(0.07- \\
0.11)\end{array}$ \\
\hline $\begin{array}{l}\text { nDBPCV } \\
\text { (95\%,interval) }\end{array}$ & $\begin{array}{l}0.16(0.09- \\
0.19) * \star \square \square\end{array}$ & $\begin{array}{l}0.05(0.02- \\
0.08)\end{array}$ & $\begin{array}{l}0.14(0.08- \\
0.17)^{\star \star}\end{array}$ & $\begin{array}{l}0.05(0.02- \\
007)\end{array}$ & $\begin{array}{l}0.13(0.07- \\
0.5)^{\star \star}\end{array}$ & $\begin{array}{l}0.04(0.02- \\
0.06)\end{array}$ & $\begin{array}{l}0.11(0.06- \\
0.14)^{\star \star}\end{array}$ & $\begin{array}{l}0.04(0.02- \\
0.05)\end{array}$ \\
\hline $\begin{array}{l}5 \text { days,n \% } \\
\text { Death rate }\end{array}$ & $6(1.875)^{\square[0 ~}$ & - & $3(0.937)$ & - & $3(1.07)$ & - & $2(0.714)$ & - \\
\hline
\end{tabular}

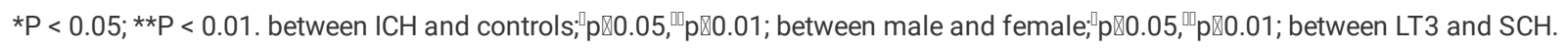

$\mathrm{ICH}$ patients and the control were divided into two gender subgroups. The BPA level of ICH men increased significantly compared with women and the control, and there was also a difference between women and the control $p<0.01$.(Figure 1 )

LT3-male nDBPcv compared with female and control, SCH male, female compared with control group, $\mathrm{P}<0.01$.(Figure 2)

Compared with the female LT3 and the control, the FT3 of the male LT3 was significantly reduced, and the female SCH group TSH was significantly increased compared with the male and the control, $\mathrm{P}<0.05$.(Figure 3)

Compared with the control, the serum BPA and protease in the LT3 and SCH groups at 15 and 30 days tended to decrease; on the contrary, the level of miR338-3p decreased, the level of miR-455 increased, and the blood pressure parameters increased. In particular, nDBPCV was more Obviously, $\mathrm{P}<0.01$. (Table 3 ) 
Table 3

Comparison of serum bisphenol A, biomarkers and death rate in different subgroups.

\begin{tabular}{|c|c|c|c|c|c|c|c|c|}
\hline 115 day & LT3 & & & & $\mathrm{SCH}$ & & & \\
\hline $\begin{array}{l}\text { Bioma bi } \\
\text { biomarer }\end{array}$ & $\begin{array}{l}\text { Male } \\
(n=240)\end{array}$ & Control $(n=240)$ & Female $(n=80)$ & Control $(n=80)$ & Male(n=80) & Control $(n=80)$ & Female(n=200) & Control $(n=200)$ \\
\hline $\begin{array}{l}\mathrm{BPA}, \mathrm{b} \text { BPA } \\
\mathrm{ng} / \mathrm{ml} \\
(2)\end{array}$ & $0.89 \pm 0.15^{\star * \square \square}$ & $0.52 \pm 0.14$ & $0.74 \pm 0.11^{\star *}$ & $0.52 \pm 0.12$ & $0.78 \pm 0.1$ ** & $0.53 \pm 0.10$ & $0.70 \pm 0.12^{\star \star}$ & $0.53 \pm 0.1$ \\
\hline $\begin{array}{l}\text { MiR-338-3p } \\
\mathrm{ng} / \mathrm{ml}\end{array}$ & $1.7 \pm 0.37 *$ & $1.21 \pm 0.2$ & $1.41 \pm 0.59 *$ & $1.22 \pm 0.55$ & $1.25 \pm 0.56$ & $1.22 \pm 0.49$ & $1.22 \pm 0.46$ & $1.21 \pm 0.49$ \\
\hline $\begin{array}{l}\text { MiR-451 } \\
\mathrm{ng} / \mathrm{ml}\end{array}$ & $3.4 \pm 0.19 *$ & $3.25 \pm 0.31$ & $3.6 \pm 0.19 *$ & $3.3 \pm 0.3$ & $3.5 \pm 0.19$ & $3.24 \pm 0.32$ & $3.45 \pm 0.19$ & $3.3 \pm 0.30$ \\
\hline $\begin{array}{l}\text { Death rate } \\
\mathrm{n}, \%\end{array}$ & $4(1.25)^{\square]}$ & - & $2(0.625)^{\square]}$ & - & $3(1.07)^{\square \square}$ & - & $1(0.357)$ & - \\
\hline 30 days & & & & & & & & \\
\hline $\begin{array}{l}\text { BPA,b } \\
\text { BPAng/ml } \\
\text { (2) }\end{array}$ & $0.89 \pm 0.14 * * \square$ & $0.52 \pm 0.12$ & $0.70 \pm 0.10$ ** & $0.52 \pm 0.11$ & $0.73 \pm 0.12^{\star \star}$ & $0.53 \pm 0.10$ & $0.66 \pm 0.10$ ** & $0.53 \pm 0.1$ \\
\hline $\begin{array}{l}\text { MiR-338-3p } \\
\mathrm{ng} / \mathrm{ml}\end{array}$ & $1.42 \pm 0.3^{\star}$ & $1.21 \pm 0.2$ & $1.28 \pm 0.59 *$ & $1.23 \pm 0.55$ & $1.24 \pm 0.56$ & $1.22 \pm 0.49$ & $1.217 \pm 0.4$ & $1.21 \pm 0.42$ \\
\hline $\begin{array}{l}\text { MiR-451 } \\
\mathrm{ng} / \mathrm{ml}\end{array}$ & $3.6 \pm 0.19$ & $3.28 \pm 0.27$ & $3.7 \pm 0.18$ & $3.26 \pm 0.31$ & $3.8 \pm 0.19$ & $3.24 \pm 0.34$ & $3.9 \pm 0.19$ & $3.3 \pm 0.35$ \\
\hline $\begin{array}{l}\text { Death rate } \\
\mathrm{n}, \%\end{array}$ & $20(6.25)^{(2000}$ & - & $8(1.56)$ & - & $7(2.5)$ & - & $6(2.14)$ & - \\
\hline
\end{tabular}

$\star \mathrm{P}<0.05 ; * \star \mathrm{P}<0.01$. between ICH and controls, $\mathrm{p} \otimes 0.05,{ }^{\square} \mathrm{p} \otimes 0.01$; between male and female; $\mathrm{p} \otimes 0.05, \mathrm{p}$

『0.01; between LT3 and SCH.

There were 600 cases of $\mathrm{ICH}$, and the recent total mortality rate was 74 cases (12.3\%). There were 320 cases of LT3 and 50 deaths (15\%); 280 cases of SCH and 24 deaths (8.57\%). The recent mortality rates of the two groups were compared in 5,15 , and 30 days.15 (4.7) vs $7(2.5), 20(6.25)$ vs $10(3.75), 15(4.68)$ vs 7(2.5) $\mathrm{P} \otimes 0.01$. (Figure 4)

On day 2, serum miR-338-3p significantly increased compared with the control, while miR-445 significantly decreased. P<0.01.(Figure 5)

On day 15 serum miR-338-3p significantly lowed compared with the control,while miR-451 significantly increased.P<0.01.(Figure 6)

Serum miR-338-3p decreased more significantly on day 30 compared with that on day 15 and the control, while miR-451 increased significantly. P<0.01.

(Figure 7)

Serum BPA is positively correlated with biomarker, 24hDBPCV, and was negatively correlated LT3 and niR-451.(Table 4)

Table 4. Linear correlation analysis of serum BPA囚ABP and biomarkers in YICH.

\begin{tabular}{|c|c|c|c|c|c|c|c|c|c|}
\hline \multicolumn{3}{|c|}{ Variables } & \multirow{2}{*}{$\begin{array}{l}\text { rvalue } \\
0.46\end{array}$} & \multirow{2}{*}{$\begin{array}{l}\text { P value } \\
₫ 0.01\end{array}$} & \multicolumn{3}{|c|}{ Variables } & \multirow{2}{*}{$\begin{array}{l}\text { rvalue } \\
0.55\end{array}$} & \multirow{2}{*}{$\begin{array}{l}\mathrm{P} \text { value } \\
₫ 0.01\end{array}$} \\
\hline BPA & and & miR-388-3p & & & BPA & and & thrombin & & \\
\hline BPA & and & 24hDBPCV & 0.48 & $\triangle 0.01$ & BPA & and & miR-451 & -0.52 & $\bowtie 0.01$ \\
\hline BPA & and & MMP-9 & 0.51 & $\otimes 0.01$ & BPA & and & FT3 & -051 & $\bowtie 0.01$ \\
\hline
\end{tabular}

The increase of nSBP and nDBP were used as dependent variables, and age, gender, BMI and BPA were used as independent variables. The results of multiple linear regression showed that cumulative BPA exposure was an independent factor affecting of nSBP and nDBP.(Table 5)

Table 5. Multiple linear regression analysis of $n S B P$ and nDBP increase $(n=600)$ 


\begin{tabular}{|cccccccc|}
\hline D variable & I variable & B & SE & $\beta$ & t value & P value & \\
\hline nSBP & constant & 16.432 & 1.939 & - & 7.482 & $<0.01$ \\
& BPA & 1.935 & 0.448 & 0.229 & 3.452 & $<0.05$ \\
\hline nDBP & constant & 18.449 & 2.439 & - & 8.442 & $<0.01$ \\
\hline & BPA & 2.335 & 0.398 & 0.286 & 4.452 & $<0.01$ \\
\hline
\end{tabular}

The relationship between BPA, thrombin and miRNA expression risk factors: draw a BPA ROS curve, the area under the curve is 0.744 (95\% $\mathrm{Cl} 0.624-0.894$ ), the maximum Uden index is 0.426 , the sensitivity is $75.4 \%$, and the specificity is $67.4 \%$, corresponding to a cut-off value of 1.567 . Adjusting for confounding factors such as gender, age, and marital status, the risk of BPA in ICH patients is 5.12 times that of the general population. (Table 6)

Table 6 Logistic regression analysis of serum bisphenol A level,blood lipid and risk factors $(n=280)$

\begin{tabular}{|c|c|c|c|c|c|c|}
\hline Independent variable & B & SE & $\mathrm{X}^{2}$ value & $P$ value & \multicolumn{2}{|c|}{ OR 95\%Cl } \\
\hline BPA & 2.86 & & 0.613 & 9.89 & 0.003 & $7.413 \varangle 0.624-0.931 \rrbracket$ \\
\hline Thrombin & 2.41 & & 0.514 & 8.99 & 0.002 & $5.114 \rrbracket 0.891-1.614 \rrbracket$ \\
\hline miR-338-3p & 1.618 & & 0.412 & 12.007 & 0.001 & $4.170(1.861-9.431)$ \\
\hline \multicolumn{7}{|l|}{ BMI stratification normal } \\
\hline Thin & -0.214 & & 0.891 & 0.059 & 0.124 & $6.784(0.129-4.718)$ \\
\hline Overweight, obese & 1.090 & & 0.412 & 6.410 & 0.011 & $2.803(1.247-6.313)$ \\
\hline Insulin resistance index & 1.214 & & 0.539 & 7.140 & 0.009 & $3.143(1.349-8.726)$ \\
\hline High triglycerides & 1.225 & & 0.397 & 5.765 & 0.02 & $2.56(1.19-4.976)$ \\
\hline High Tch & 1.231 & & 0.497 & 6.476 & 0.01 & $2.948(1.241-5.736)$ \\
\hline
\end{tabular}

Increase BPA levels (none $=0$, yes $=1$ ) and miR-451 (none $=0$, yes $=1$ ), nDBPCV (none $=0$, yes $=1$ ), binding and interaction analysis. The results of the crosshealth analysis showed that after adjusting for factors such as gender, age, and marital status. Nocturnal hypertension and the low expression of miR144/451 and the presence of elevated serum BPA are significantly different from the BPA levels of patients with non-nocturnal hypertension, and there is a risk of ICH. The results of interaction analysis showed that there is a multiplicative and additive interaction between whether serum BPA is related to nocturnal hypertension $(U=4.19, P<0.05)$ and whether miR-451 is under-expressed $(U=6.16, P<0.05) \otimes T a b l e ~ 7 \rrbracket$

Table 7 Effect of BPA level on the coefficient of variation of night blood pressure $(n=280)$

\begin{tabular}{|c|c|c|c|c|c|c|}
\hline \multicolumn{3}{|c|}{-BPA miR-451 nDBPCV } & \multirow{2}{*}{$\begin{array}{l}\text { NBP } \\
\text { (n) }\end{array}$} & \multirow{2}{*}{$\begin{array}{c}\text { OR } \\
(95 \% \mathrm{CI})\end{array}$} & \multirow[t]{2}{*}{ MI } & \multirow[t]{2}{*}{ AI } \\
\hline & (n) & (n) & & & & \\
\hline No & No & No & 240 & $1.947(0.175-29.987)$ & 0.0134 & RERI:3.12(0.912-3.123) \\
\hline No & No & No & 1 & $0.765(0.32-0.915$ & & AP:5.13 ( 0.723-1.346) \\
\hline Yes & 160 & 240 & 0 & $4.997(1.845-9.365)$ & & $\mathrm{S}: 1.715(0.541-15.57)$ \\
\hline Yes & 140 & 240 & 0 & $19.778(1.879-245.667)$ & & \\
\hline
\end{tabular}

\section{Discussion}

Bisphenol A (BPA) is an endocrine disruptor, which not only interferes with thyroid function, but also affects sexual function. Studies have reported $\left.{ }^{[12-14}\right]_{\text {that }}$ in stress diseases such as heart and brain, female patients are mostly at risk of SCH This study found that the serum BPA in the male group of YICH patients was significantly increased compared with the female group, and the free testosterone (FT) level in men was significantly reduced. It also shows that women 
are mostly SCH, while men are mostly LT3 syndrome. It is speculated that there is a certain inherent correlation between the toxic effects of BPA exposure on the thyroid and sexual function, involving a chain of chain events.

Ambulatory blood pressure is related to target organ damage, and the circadian rhythm of blood pressure has an important influence on the pathophysiology of diseases. Studies have reported that ${ }^{[15-17]}$ BPA exposure from canned food can increase blood pressure, and blood pressure variability is involved in the occurrence and development of target organ damage ${ }^{[18]}$. Although BPA exposure does not directly affect plasma lipid levels, it can enhance the expression of CD36 in macrophages, which is related to lipid accumulation ${ }^{[19]}$. There are changes in oxidized protein and lipid levels in atherosclerosis ${ }^{[20]}$. The baseline data of this study showed that the blood lipid level of $\mathrm{YICH}$ patients was significantly higher than that of the control, and the night diastolic blood pressure and blood pressure scores were significantly increased. In addition, multiple linear regression analysis showed that BPA is an independent risk factor that affects the percentage increase of nSBP and nDBP.

The biological trigger BPA is biochemically toxic to multiple organ functions. Exposure is related to oxidative stress, insulin resistance, and changes in microRNA (miR) expression profiles ${ }^{[21,22]}$, defining the potential mechanism of toxic miR. miR-144/451 may be beneficial under stress conditions and has a certain protective effect on vascular endothelial function. Oxidative stress is a series of reactive oxidation, forming a series of network links from biological toxicity to cytotoxicity and lipotoxicity.

Inflammation and apoptosis are related to the prognosis and survival of patients, and the inflammatory environment can cause neuronal apoptosis ${ }^{[23]}$. BPA exposure can induce vascular toxicity and risk events, which can gradually affect cerebrovascular pathology and molecular phenotypic biochemical factors. Nuclear factor-KB transcription factor is a multifunctional transcription factor. The NF-KBp65 inflammatory pathway induces oxidative stress and apoptosis, and improves cytological functions ${ }^{[24]}$. Inflammation, oxidative stress and other factors can cause vascular dysfunction and structural damage ${ }^{[25]}$. Therefore, targeting TLR4 in ICH patients can attenuate the inflammatory response, thereby attenuating apoptosis and improving prognosis ${ }^{[26]}$. The study of this group showed that serum inflammatory cytokines and TLR4-NF-KB signaling pathway series factors were significantly increased, and the initial thrombin concentration was associated with a higher risk after stroke, indicating that the expression of ncRNA has time-point changes. It is speculated that this phenomenon is related to the treatment and the protective factors of the body's autoimmunity. This study also found that the recent mortality rate of the LT3 group was significantly increased compared with that of the $\mathrm{SCH}$ group, indicating the resonance of the body, which needs to be confirmed by further studies. In addition, correlation analysis showed that BPA was positively correlated with miR-338-3P and a variety of proteases, and negatively correlated with miR451.

The main limitation of this study is the lack of analysis and research on the relationship between the drug and BPA biotoxicity and biomarkers. In-depth analysis of the anti-inflammatory, network homeostasis and neuroprotective effects of connexin 43 after ICH injury is required. Clarify the innervation or position of $\beta 3$ adrenergic nerves, and target the adrenergic system to effectively inhibit the neuroinflammatory mechanism after ICH

\section{Conclusion}

High BPA exposure strongly predicts the risk of $\mathrm{YICH}$ patients. Activation of NF-KB signaling pathway, secondary thyroid function and ABP changes, together to construct an inflammatory response program, is related to the risk of short-term death.

\section{Declarations}

\section{Availability of data and materials}

The de-identified dataset generated and analyzed in this study is electronically stored and protected by specific password. The dataset is available from the corresponding author on reasonable request.

\section{Acknowledgements}

Thanks to Professor Wu Bowei ,Zhang Mingshen and some teachers, residents, students of Shanxi Medical University

\section{Funding}

This work was supported by the Natural Science Foundation of Shanxi Province (grant no. 201701D121177); Shanxi Science,Technology Department of the Project Fund (2011099); Spark Planning Project of Changzhi (200704009).

\section{Author contributions}

Dr GPW responsible for the writing of the first draff and collection and preservation of some specimens; Prof,DG undertake specimen testing and data analysis;Drs LF Z,LL Z, AVW,CPF recruited eligible patients and collected the blood sample;Drs PG, undertook to follow up ;Dr WLS recruited and analysis CT and MRI data;all authors contribute to the subsequent draft ;Dr GPW,JYL,ZDQ and Drs JHC, conducted study design and manuscript revisio.

\section{Corresponding author}

Correspondence to guoping wang. 


\section{Ethics declarations}

\section{Ethics approval and consent to participation}

All methods in this study were performed in accordance with the Declaration of Helsinki and approved by the Ethics Committee of Changzhi People's Hospital affiliated with Shanxi Medical University (No: 2013093).All data obtained from the Changzhi research database have been de-identified, and all patients have the right to know the project and sign an informed consent form.

\section{Consent for publication}

Not applicable.

\section{Competing interests}

The authors declare that there was no conflicts of interest.

\section{References}

1. Lewis B.Morgenstern, J.Claude HemphillIII,, Caraig Anderson, Kyra. Becker, Joseph P..Broderick,E..Sander.Connolly J.r,Steven M..Greenberg, James N.Huang,R.\&.Loch Macdonald. Guidelines for the management of spontaneous intracerebral hemorrhage: A guideline for healthcare professionals from the American Heart Association/American Stroke Association. Stroke. 2010,41: 2108-2129.doi.10. 1161/ STR.0b 013e 3181ec611b.

2. Valery L. Feigin, Grant Nguyen, \& Kelly Cercy, B.S.,.Global, Regional, and Country-Specific Lifetime Risks of Stroke, 1990 and 2016. The GBD 2016 Lifetime Risk of Stroke Collaborators.N Engl J Med.2018; 379:2429-2437.doi.10.1056/ NEJMoa 1804492

3. Louise V. Wain,Ahmad Vaez,Rick Jansen,Roby Joehanes,Peter J. van der Most,A. Mesut Erzurumluoglu,PaulF.O'Reilly,Claudia P. Cabrera.Novel Blood Pressure Locus and Gene Discovery Using Genome-Wide Association Study and Expression Data Sets From Blood and the Kidney.Hypertension.2017;70:e4-e19./doi.10.1161/HYPERTENSIO NAHA.117. 09438.

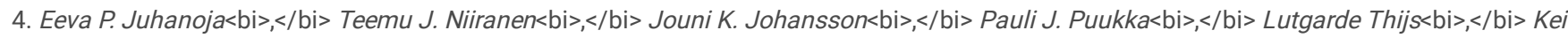

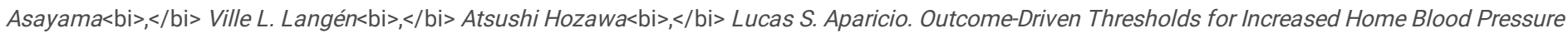
Variability.Hypertension. 2017;69(4):599-607<bi>.</bi>https://doi.org/10.1161/HYPERTENSIONAHA.116.08603.

5. Samuel X.Shil, Kaibin Shil,and Qiang Liu.Brain injury instructs bone marrow cellular lineage destination to reduce neuroinflammation.Science Translational Medicine.2021;13 (589):eabc7029.doi.10.1126/scitranslmed.abc7029.

6. Warner, G.R., Flaws, J.A. Bisphenol-A and phthalates: How environmental chemicals are reshaping toxicology. Toxicol Sci.2018;166:246-249.doi. 10.1093 / tox sci/ kfy232.

7. Roberto Bono,Valenia Bellisario,Roberta Tassinari,Giulia Squillacioti,Tilde Manetta, Massimiliano Bugiani, Enrica Migliore and Pavilio Piccioni.Bisphenol A,Tobacco Smoke,and Age as predictors of Oxidative Stress in Childen and Adolescents.Int J.Environ Res Public Health.2019;16, 20252037.doi.org/10.3390/ijerph16112025.

8. Kharrazian, D. The potential roles of bisphenol A pathogenesis in autoimmunity. Autoimmune Dis. 2014;2014:743616.doi:10.1155/2014/743616.

9. Provvisiero, D.P., Pivonello, C., Muscogiuri, G., Negri, M., de Angelis, C.,\& Simeoli, C.,. Influence of bisphenol A on Type 2 Diabetes Mellitus. Int J Environ Res Public Health. 2016;13:989-998 .doi.10.3390/ijerph13100989.

10. J Claude Hemphill III, Steven M Greenerg, Craig S Anderson, Kyra Becker,Bernard R Bendok, Mary Cushman, Gordon, Fung, Joshua N, Goldstein, R Loch Macdonald, Magdy H Selim,\& Danic Woo, Guideline for the Management of Spontaneous Intracerebral Hemorrhage. Stroke.2015;46:2032- 2060.doi.10. 1161/STR.000000 0000000069.

11. 1999 World Health Organization-International Society of Hypertension Guidelines for the Management of Hypertension.Clinical and Experiment Hypertension. 2009; 21:1009- 1060 151- 183.doi.10.3109/10641969909061028.

12. Romano, M.E., Webster, G.M., Vuong, A.M., Thomas Zoeller, R., Chen, A.,\& Hoofnagle, A.N, Gestational urinary bisphenol A and maternal and newborn thyroid hormone concentrat- ions. Environ Res. 2015;138, 453-460. doi.10.1016/j.envres.2015.03.003.

13. Habtamu Azene Tekle,1 Tesfahun Molla Bobe,1 Efrata Girma Tufa, 2 and Fithamlak Bisetegen Solomon.Age-sex disparities and sub-clinical hypothyroidism among patients in Tikur Anbesa Specialized Hospital, Addis Ababa, Ethiopia.J Health Popul Nutr. 2018;37: 182018 doi:10.1186/s41043018-0149-x.

14. Sanghyuk Bae and Yun-Chul Hong. Exposure to Bisphenol A From Drinking Canned Beverages Increases Blood Pressure. Hypertension.2015;.65:313319.doi.10.1161/HYPERT- ENSIONAHA.114.04261.

15. Leoncini G,Viazzi F,Storace G,et al.Blood pressure variability and multiple organ damage in primary hypertensin [J] J Hum Hypertension,2013;27:663670.doi.org/nature. com/articles/jhh201345.

16. Izzo, R., Mancusi, C, de Simone, G. Are we underestimating prehypertension? Hyper tension. 2019;73: 541-542.doi.10.1161/HYPERTENSIONA HA. 118.12310

17. Lee, H.S., Park, Y. Identification of metabolic pathways related to the bisphenol A induced adipogenesis in differentiated murine adipocytes by using RNA sequencing. Environ Res. 2019;171, 161-169. doi.10.1016/j.envres.2019.01.017.

18. Witting, P.K., Pettersson, K.,\& Letters, J., Stocker, R. Site-specific antiatherogenic effect of probucol in apolipoprotein E-deficient mice.Arterioscler Thromb Vasc Biol.2000;20: E26- E33. doi.10.1161/01.ATV.20.8.e26. 
19. Roberto Bono,Valenia Bellisario,Roberta Tassinari Bisphenol A,Tobacco Smoke,and Age as predictors of oxidative Stress in Childen and Adolescents. Int J.Environ Res Public Health. 2019;16: 2025-2037. doi: 10.3390/ijerph1611.

20. Cetewayo S. Rashid, Amita Bansal and Rebecca A. Simmons.Paternal bisphenol A exposure alters offspring glucose tolerance in a time, dose, and sexspecific manner. Diabetes.2018; 67 (Supplement 1): 1363-P.doi.org/10.2337/db18-1363-P.

21. Minatoya, M., Araki, A., Miyashita, C., Ait Bamai, Y., Itoh, S., \& Yamamoto, J, Association between prenatal bisphenol A and phthalate exposures and fetal metabolic related biomarkers: The Hokkaido study on Environment and Children's Health. Environ Res. 2018;161, 505-

511.doi.10.1016/j.envres.2017.11.052.

22. Wei Ying,Matthew Riopel,Gautam Bandyopadhyay.Yi Dong,Amanda Birm ingh -am,Jong Bae Seo.Adipose Tissue Macrophage-Derived Exosomal miRNAs Can Modulate In Vivo and In Vitro Insulin Sensitivity.Cell.2017;171:372-384.doi.10.1016/j.cell. 2017. 08.035.

23. Xiaowei Fei, Yeting He, Jia Chen, Weitao Man, Chen Chen, Kai Sun, Boyun Ding, Chongwu Wang ,Ruxiang Xu.The role of Toll-like receptor 4 in apoptosis of brain tissue after induction of intracerebral hemorrhage.Journal of Neuroinflammation. 2019; 16: 234. DOI: 10.1186/s12974-019-1634-x.

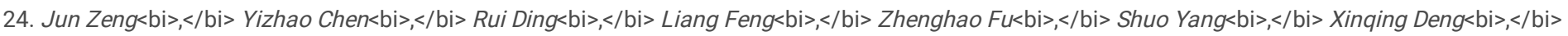
Zhichong Xie, Shizhong Zheng.Isoliquiritigenin alleviates early brain injury after experimental intracerebral hemorrhage via suppressing ROS- and/or NF$K B$-mediated NLRP3 inflammasome activation by promoting Nrf2 antioxidant pathway.Journal of Neuroinflammation<bi $></ \mathrm{bi}>2017<\mathrm{bi}>;</ \mathrm{bi}>14<\mathrm{bi}>$ : $</$ bi $>119<\mathrm{bi}>$. doi:</bi>10.1186/ s12974-017-0895-5<bi>.</bi>

25. Yang, A.L., Cui, H.-J., Zhou, H.-J.,\& Tang, T. Expression of ECM-associated genes in rat brain following experimental intracerebral hemorrhage.Stroke.2014; 45:ATP229 Suppl 1.

26. Marc Durocher, Bradley P. Ander, Glen Jickling, Farah Hamade, Heather Hull, Bodie Knepp, Da Zhi Liu, Xinhua Zhan, Anh Tran, Xiyuan Cheng, Kwan Ng, Alan Yee, Frank R. Sharp and Boryana Stamova. Inflammatory, regulatory, and autophagy co-expression modules and hub genes underlie the peripheral immune response to human intracerebral hemorrhage<bi>.</bi> Journal of Neuroin flammation. 2019;16:56.doi: 10.1186/s12974-019-1433-4.

\section{Figures}

Figure 1. Serum BPA levels in YICH patients and controls,as well as in male and

female subgroups..( $\mathrm{a}, \mathrm{b})$

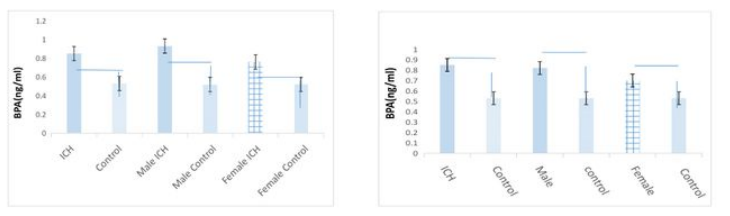

Figure 1

Serum BPA levels in YICH patients and controls,as well as in male and female subgroups..( $a, b)$

Page $11 / 17$ 
Figure 2. nDBPCV levels in YUICH and control

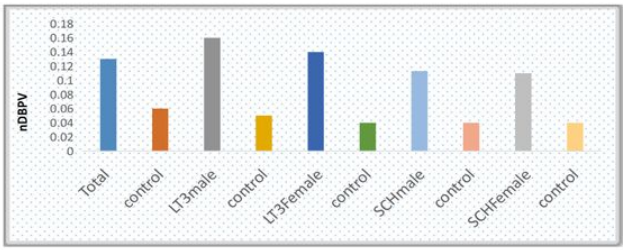

Figure 2

nDBPCV levels in YUICH and control 
Figure 3 :Comparative analysis of $\mathrm{ICH}$ thyroid function of different genders and control

group $(a, b)$
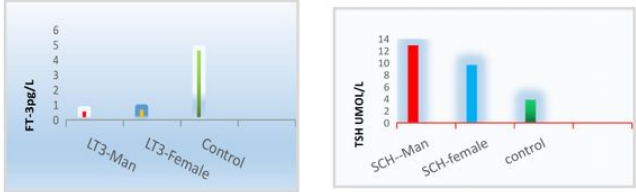

Figure 3

Comparative analysis of $\mathrm{ICH}$ thyroid function of different genders and control group $₫ a, b \rrbracket$ 
Figure 4: Comparison of mortality between the LT3 and SCH groups

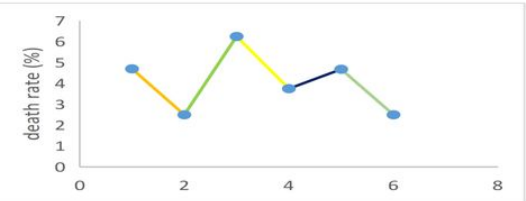

Figure 4

Comparison of mortality between the LT3 and $\mathrm{SCH}$ groups 
Figure 5, Comparison of miR-338-3p ,miR-451 levels in the two groups ( 2 days. a,b, c,d)
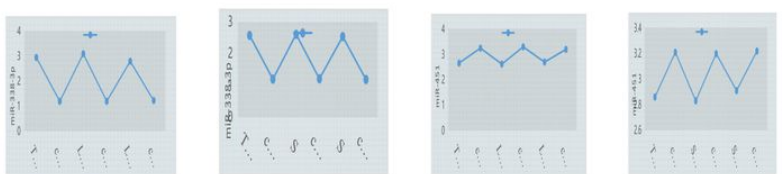

Figure 5

Comparison of miR-338-3p ,miR-451 levels in the two groups (2 days. a,b, c,d囚 
Figure 6 : Comparison of serum miR-338-3p and miR-451 levels in the two groups

(For 15, a,b,c,d)

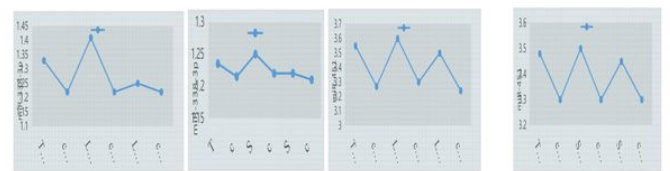

Figure 6

Comparison of serum miR-338-3p and miR-451 levels in the two groups $\otimes$ For $15 . a, b, c, d \rrbracket$ 
Figure 7 : Comparison of serum miR-338-3p and miR-451 levels in the two groups

(For $30 .-\mathrm{a}, \mathrm{b}, \mathrm{c}, \mathrm{d}$ )
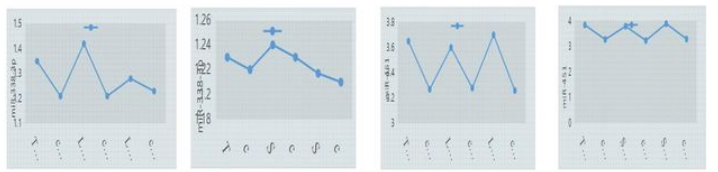

Figure 7

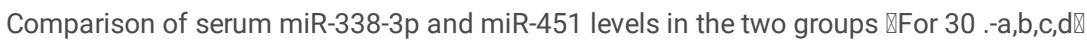

\section{Supplementary Files}

This is a list of supplementary files associated with this preprint. Click to download.

- BPA2.doc

- childreninformed3.doc

- informedconsent4.doc 